\title{
SYSTEMS OF ELLIPTIC EQUATIONS INVOLVING MULTIPLE INVERSE-SQUARE POTENTIALS AND CRITICAL EXPONENTS
}

\author{
DONGSHENG KANG AND XIAOFENG SHEN
}

Abstract. In this paper, a system of elliptic equations is investigated, which involves multiple critical Sobolev exponents and singular points. The best Sobelev constant related to the system is studied, which is verified to be independent of the location of singular points. By a variant of the concentration compactness principle and the mountain-pass argument, the existence of positive solutions to the system is proved. At last, the existence of sign-changing solutions to the system is also established on the basis of the mountain-pass-type positive solutions.

Mathematics subject classification (2010): 35B33, 35J60.

Keywords and phrases: elliptic system, solution, critical exponent, Hardy inequality, variational method.

\section{REFERENCES}

[1] B. Abdellaoui, V. Felli, I. Peral, Existence and nonexistence for quasilinear equations involving the p-Laplacian, Boll. Unione Mat. Ital. Sez. B, 9 (2006), 445-484.

[2] B. AbDellaoui, V. Felli, I. Peral, Some remarks on systems of elliptic equations doubly critical in the whole $\mathbb{R}^{N}$, Calc. Var. Partial Differential Equations, 34 (2009), 97-137.

[3] A. Ambrosetti, H. Rabinowitz, Dual variational methods in critical point theory and applications, J. Funct. Anal., 14 (1973), 349-381.

[4] M. Bouchekif, Y. NAsRi, On a singular elliptic system at resonance, Ann. Mat. Pura. Appl., 189 (2010), 227-240.

[5] H. Brezis, L. Nirenberg, Positive solutions of nonlinear elliptic equations involving critical Sobolev exponents, Comm. Pure Appl. Math., 36 (1983), 437-477.

[6] D. CAO, P. HAN, Solutions to critical elliptic equations with multi-singular inverse square potentials, J. Differential Equations, 224 (2006), 332-372.

[7] F. CATRINA, Z. WANG,On the Caffarelli-Kohn-Nirenberg inequalities: sharp constants, existence (and nonexistence), and symmetry of extremal functions, Comm. Pure Appl. Math., 54 (2001), 229258.

[8] G. Cerami, S. Solimini, M. Struwe, Some existence results for superlinear elliptic boundary value problems involving critical exponents, J. Funct. Anal., 69 (1986), 289-306.

[9] H. EGNELL, Elliptic boundary value problems with singular coefficients and critical nonlinearities, Indiana Univ. Math. J., 38 (1989), 235-251.

[10] V. FELLI, S. TERRACINI, Elliptic equations with multi-singular inverse-square potentials and critical nonlinearity, Comm. Partial Differential Equations, 31 (2006), 469-495.

[11] D. Figueiredo, I. Peral, J. Rossi, The critical hyperbola for a Hamiltonian elliptic system with weights, Ann. Mat. Pura Appl., 187 (2008), 531-545.

[12] N. Ghoussoub, C. YuAn, Multiple solutions for quasi-linear PDEs involving the critical Sobolev and Hardy exponents, Trans. Amer. Math. Soc., 352 (2000), 5703-5743.

[13] G. Hardy, J. Littlewood, G. Polya, Inequalities, reprint of the 1952 edition, Cambridge Math. Lib., Cambridge University Press, Cambridge, 1988.

[14] Y. HuAnG, D. KANG, Elliptic systems involving the critical exponents and potentials, Nonlinear Anal., 71 (2009), 3638-3653.

[15] Y. HUANG, D. KANG, On the singular elliptic systems involving multiple critical Sobolev exponents, Nonlinear Anal., 74 (2011), 400-412. 
[16] E. JANnELLI, The role played by space dimension in elliptic critcal problems, J. Differential Equations, 156 (1999), 407-426.

[17] D. KANG, Concentration compactness principles for the systems of critical elliptic equations, Differ. Equ. Appl., 4 (2012), 435-444.

[18] D. KANG, Y. HuAng, S. LiU, Asymptotic estimates on the extremal functions of a quasilinear elliptic problem, J. South-Central Univ. Natl. Nat. Sci. Ed., 27(3) (2008), 91-95.

[19] P.L.Lions, The concentration compactness principle in the calculus of variations, the limit case (I), Rev. Mat. Iberoamericana, 1(1) (1985), 145-201.

[20] P. L. Lions, The concentration compactness principle in the calculus of variations, the limit case(II), Rev. Mat. Iberoamericana, 1(2) (1985), 45-121.

[21] Z. LiU, P. HAN, Existence of solutions for singular elliptic systems with critical exponents, Nonlinear Anal., 69 (2008), 2968-2983.

[22] Z. TANG, Sign-changing solutions of critical growth nonlinear elliptic systems, Nonlinear Anal., 64 (2006), 2480-2491.

[23] S. TERRACINI, On positive entire solutions to a class of equations with a singular coefficient and critical exponent, Adv. Differential Equations, 2 (1996), 241-264.

[24] J. L. VAZQUEZ, A strong maximum principle for some quasilinear elliptic equations, Appl. Math. Optimization, 12 (1984), 191-202.

[25] M. Willem, Minimax Theorems, PNLDE 24, Birkhäuser, Boston, Basel, Berlin, 1996.

[26] M. Willem, Analyse fonctionnelle élémentaire, Cassini Éditeurs, Paris, 2003. 